\title{
Crystal structure of the C47S mutant of human peroxiredoxin 5
}

\author{
Christine Evrard ${ }^{(1)}$ Aude Smeets, ${ }^{(1)}$ Bernard Knoops, ${ }^{(2)}$ and Jean-Paul Declercq ${ }^{(1)}$ \\ (1) Unit of Structural Chemistry (CSTR), Université catholique de Louvain, 1 place Louis Pasteur, B-1348 Louvain-la-Neuve, Belgium. \\ ${ }^{(2)}$ Laboratory of Cell Biology, Institut des Sciences de la Vie, Université catholique de Louvain, 5 place Croix du Sud, B-1348 Louvain-la- \\ Neuve, Belgium.
}

\begin{abstract}
In the crystal structure of the reduced form of the wild-type human peroxiredoxin 5 , the presence of a benzoate ion in direct interaction with the peroxidatic cysteine (Cys 47) appeared as a rather intriguing feature since it is known that the benzoate ion can play the role of a specific hydroxyl radical scavenger. The crystal structure of the C47S mutant of the same enzyme has been crystallized in the tetragonal system, space group $P 4_{1} 22_{1} 2$, with $a$ $=65.65 \AA, c=122.04 \AA$. It confirms the presence of this benzoate ion in spite of the mutation into a serine of the Cys 47 residue to which the benzoate ion was directly linked in the wild-type structure. The benzoate ion seems to be stabilized by hydrophobic contacts on both sides of the aromatic ring. In this matter, the $\alpha 5$ helix, which is specific to peroxiredoxin 5 among mammalian peroxiredoxins, plays an important role. These hydrophobic contacts also allow to suggest why the benzoate ion disappears when the molecule is oxidized.
\end{abstract}

Keywords : Antioxidant enzyme ; peroxiredoxin ; thioredoxin fold ; thioredoxin peroxidase.

\section{INTRODUCTION}

The peroxiredoxins (PRDXs) define a large family of peroxidases able to reduce hydrogen peroxide and alkyl hydroperoxides from prokaryotes to eukaryotes. ${ }^{1-3}$ Functionally, PRDXs have been implicated in antioxidant protection and regulation of redox-dependent signaling in cells. Structurally, all PRDXs contain a conserved peroxidatic Cys residue in the $\mathrm{N}$-terminal portion of the protein, that attacks the peroxide and is oxidized to Cys sulfenic acid. PRDXs are classified into three subgroups depending on the number of Cys residues directly involved in catalysis and on the resolution mechanism of the Cys sulfenic acid. PRDX1-PRDX4 represent the typical 2-Cys mammalian subgroup and are obligate homodimers. In this subgroup, the N-terminal Cys residue (Cys51 in human PRDX2) oxidized to sulfenic acid is attacked by the resolving Cys (Cys 172 in human PRDX2) located in the C-terminus of the other subunit resulting in the formation of an intermolecular disulfide bond. PRDX5 is the only identified mammalian member of the atypical 2-Cys subgroup. In atypical 2-Cys PRDXs, the C-terminal resolving Cys is contained in the same protein and the reaction with the peroxidatic Cys results in the formation of an intramolecular disulfide bond. Finally, PRDX6 is classified in the mammalian 1-Cys subgroup in which the $\mathrm{N}$-terminal peroxidatic Cys (Cys47) is conserved and the resolving C-terminal Cys is missing.

Crystal structures are known for members of the typical 2-Cys subgroup (PRDX1, ${ }^{4}$ PRDX2 $2,5,6$ ). PRDX6 represents the mammalian 1-Cys subgroup for which a crystal structure is also available. ${ }^{7}$ In typical 2-Cys and 1Cys subgroups, the crystal structures are characterized by the formation of tightly associated dimers resulting from the association of the $\beta$ sheets of two monomers to form a 10- or 14 -stranded $\beta$-sheet. PRDX5, the mammalian atypical 2-Cys PRDX, was characterized ${ }^{8,9}$ and the structure of its reduced state was recently solved by our group in two different crystal forms. ${ }^{10,11}$ Another crystal form simultaneously containing the reduced state and an unexpected dimeric oxidized state was also described. ${ }^{12}$ All the reduced molecules show that the sulfur atoms of Cys47 and Cys151 are positioned too far apart (13.8 $\AA$ ) to interact in the expected intramolecular way without large conformational changes. The dimer present in all other known PRDXs structures and resulting from the association of $\beta$ sheets is not observed in PRDX5. Also, the crystal structure of the reduced form of PRDX5 allowed to discover the presence of a benzoate ion, a specific scavenger of hydroxyl radical, ${ }^{13}$ covering the active site pocket and interacting with the sulfur atom of the conserved peroxidatic cysteine residue (Cys47).

Since the mutation of the peroxidatic Cys47 into a Ser residue (C47S) in human PRDX5 inhibits its peroxidase activity $^{9}$ and its antioxidant protection in vitro and in vivo, ${ }^{14}$ and since benzoate ion known as a scavenger of hydroxyl radical was directly linked to Cys 47 residue in the wild-type structure, additional structural 
investigations were necessary to determine whether a loss of antioxidant activity of human PRDX5 C47S mutant could only be due to the replacement of the catalytic Cys by a Ser or also to conformational changes leading to the release of the benzoate ion.

Here we present the crystal structure analysis of human PRDX5 C47S mutant. The crystal structure refined at 1.7 $\AA$ shows the presence of the benzoate ion in spite of the mutation of Cys47 into a Ser. The benzoate ion appears to be stabilized by hydrophobic contacts on both sides of the aromatic ring.

\section{EXPERIMENTAL SECTION}

The expression and purification of wild-type human PRDX5 and mutant C47S were previously described. ${ }^{10,14}$ The crystals of the 6 xHis-tagged molecule were grown in reducing conditions by hanging or sitting drop vapour diffusion at $18^{\circ} \mathrm{C}$ with the well solution $(500 \mu \mathrm{L})$ consisting of $1.6 \mathrm{M}$ ammonium sulfate and $0.2 \mathrm{M}$ potassium sodium tartrate as precipitant, $0.1 \mathrm{M}$ sodium citrate buffer $\mathrm{pH} 5.3,10^{-3} \mathrm{M}$ 1,4-dithio-dl-threitol as antioxidant and $0.02 \%(\mathrm{w} / \mathrm{v})$ sodium azide. The crystallization drop was formed by mixing equal amounts $(2-10 \mu \mathrm{L})$ of the protein solution $\left(10 \mathrm{mg} \mathrm{mL}^{-1}\right)$ and of the well solution.

Before data collection, the crystal was cryo-soaked for a few seconds in paratone and flash cooled at $100 \mathrm{~K}$. Data were collected at $1.7 \AA$ resolution on the synchrotron BW7A beam line at EMBL c/o DESY using a MAR-ccd detector. The images were processed with the program DENZO ${ }^{15}$ and merged with the program SCALEPACK. ${ }^{15}$ The crystals are tetragonal, space group $P 4_{1} 2{ }_{1} 2$, with $a=65.65 \AA, c=122.04 \AA$, and one protein molecule in the asymmetric unit. Five percent of the reflections were flagged for use in $R_{\text {free }}$ calculations. ${ }^{16}$ Some statistics of data collection and processing are presented in Table 1.

Since the space group and the cell dimensions of the C47S mutant of PRDX5 allowed to suppose a probable isomorphism with the tetragonal structure of the wild-type enzyme ${ }^{10}$ ( $\mathrm{pdb}$ entry $1 \mathrm{hd} 2$ ), these coordinates were used for starting the refinement after applying the appropriate mutation. A rigid body refinement using REFMAC $5^{17}$ belonging to the CCP4 suite ${ }^{18}$ yielded values of 0.343 and 0.348 for $R$ and $R_{\text {free }}$, respectively. Some minor adjustments were performed with $\mathrm{O}^{19}$ and were followed by cycles of refinement with REFMAC5. The Nterminal His tag and the short linker were not observed except for the last residue (Ser 0). Electron density for a benzoate ion was apparent in the initial electron density map despite its absence in the initial model used for phasing. Solvent molecules were progressively introduced by ARP/wARP. ${ }^{20}$ A significant improvement was reached by refining the mean square displacements of the unique molecule considered as a rigid body and defined as a TLS group. It was necessary to introduce alternate conformations for a number of side chains, mainly located at the surface of the molecule: Glu13, Glu18, Asn21, Glu27, Glu57, Gln68, Ser74, Glu83, Lys89, Glu91, Asp114, Ser159. The final $R$ value is $0.146\left(R_{\text {free }}=0.169\right)$ for a model containing 3 chloride ions and 285 solvent molecules.

Table 1. Statistics of Data Collection and Refinement Wavelength $(\AA)$

Resolution $(\AA)$

Overall (ov)

$37-1.70$

Measured reflections

Highest shell (hs)

Unique reflections

Completeness (\%)

Rsym

$\langle I / \sigma(I)\rangle$

Refinement

$R$ factor $\left(R_{\text {free }}\right)$

Estimated overall coordinate error $(\AA)$

r.m.s. deviations from ideality

r.m.s. deviations in $B$ values (bonded atoms) $\left(\AA^{2}\right)$

Mean $B$ values $\left(\AA^{2}\right)$ before TLS refinement

$\begin{array}{cc}\text { Overall (ov) } & 37-1.70 \\ \text { Highest shell (hs) } & 1.74-1.70 \\ & 184,830 \\ \text { ov/hs } & 30,149 \\ \text { ov/hs } & 99.8 / 100 \\ \text { ov/hs } & 0.053 / 0.292 \\ & 32 / 5.5 \\ \text { Overall } & \\ \text { Highest shell } & 0.146(0.169) \\ \text { Based on } R_{\text {free }} & 0.179(0.171) \\ \text { Based on maximum likelihood } & 0.068 \\ \text { Bonds }(\AA) & 0.040 \\ \text { Angles }\left({ }^{\circ}\right) & 0.019 \\ \text { Main chain } & 1.63 \\ \text { Side chain } & 0.82 \\ \text { Main-chain atoms } & 2.44 \\ \end{array}$




\section{RESULTS AND DISCUSSION}

All the 161 residues of the protein are well defined in the electron density. As shown in Fig. 1, the overall structure is characterized by the presence of a thioredoxin fold consisting of a four-stranded $\beta$ sheet and three flanking $\alpha$ helices $^{23}$ and the benzoate ion remains present at the surface of the active site pocket. Each molecule comprises three additional $\alpha$ helices and three $\beta$ strands, one of which is associated with the thioredoxin $\beta$ sheet to form a fifth strand while the two remaining ones form an additional two stranded $\beta$ sheet in the N-terminal part of the molecule. Since the overall folding is very similar to that described for the two crystal forms of the wild PRDX5 structures, ${ }^{10,11}$ it will not be further discussed.

In the tetragonal (1hd2) structure of the reduced wild-type PRDX5 enzyme, the presence of a benzoate ion located at the surface of the active site pocket ${ }^{10}$ appears as a completely unexpected structural feature. This ion was also found in the monoclinic structure $(1 \mathrm{~h} 4 \mathrm{o})^{11}$ for each of the eight independent molecules of the asymmetric unit. As benzoate is known to be a specific hydroxyl radical scavenger, its presence close to the active site might not be fortuitous. The present study confirms the presence of the benzoate ion (Fig. 1) in the C47S mutant. In lhd2, the distance between the oxygen atom $\mathrm{O} 1$ of the benzoate ion and the sulfur atom of Cys47 is $3.4 \AA$ and we now observe a distance of $2.92 \AA$ with the $\mathrm{O}^{\gamma}$ atom of Ser47, confirming the presence of a hydrogen bond between the two oxygen atoms. Other distances involving the carboxylate oxygen atoms of the benzoate ion are compared in Table $2 \mathrm{a}$ and show that in the C47S mutant this part of the benzoate is even better anchored to the active site pocket than in the wild-type enzyme.

Fig. 1. The structure of the C47S mutant of human peroxire-doxin 5. The structural elements representative of the thiore-doxin fold are colored green (helices) and red (sheets). The benzoate ion and the side chains of some important residues discussed in the text are represented. Figure prepared using MOLSCRIPT ${ }^{2 I}$ and RASTER3D. ${ }^{22}$

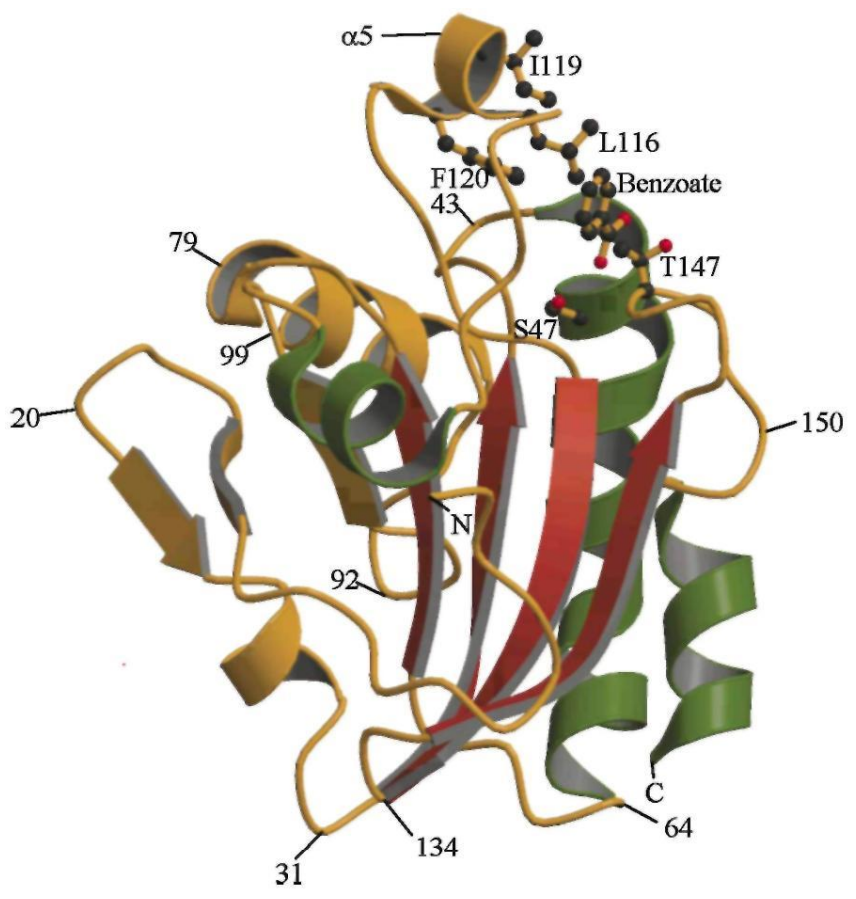


Table 2. Distances $(\AA)$ Involving Atoms of the Benzoate Ions

\begin{tabular}{|c|c|c|c|}
\hline \multirow{2}{*}{\multicolumn{4}{|c|}{ (a) Distances involving the carboxylate oxygen atoms }} \\
\hline & & & \\
\hline \multirow[t]{5}{*}{ Benzoate $\mathrm{O}^{1}$} & Thr44 $\mathrm{O}^{\gamma 1}$ & 3.07 & 3.38 \\
\hline & Gly46 N & 3.26 & 3.01 \\
\hline & Cys/Ser47 N & 2.98 & 3.19 \\
\hline & Cys $S^{\gamma} / \operatorname{Ser} 47 \mathrm{O}^{\gamma}$ & 2.92 & 3.40 \\
\hline & $\operatorname{Arg} 127 \mathrm{~N}^{\eta 2}$ & 2.79 & 2.99 \\
\hline Benzoate $\mathrm{O}^{2}$ & Gly46 N & 2.67 & 2.68 \\
\hline \multicolumn{4}{|c|}{ (b) Hydrophobic contacts involving the aromatic ring } \\
\hline Benzoate $\mathrm{C}^{3}$ & Ile119C $\mathrm{C}^{\delta 1}$ & 4.70 & 4.74 \\
\hline \multirow[t]{2}{*}{ Benzoate $\mathrm{C}^{4}$} & Leu116C $C^{\delta 1}$ & 3.99 & 3.87 \\
\hline & Phe120 $C^{\zeta}$ & 3.57 & 3.73 \\
\hline Benzoate $\mathrm{C}^{5}$ & $\operatorname{Thr} 147 \mathrm{C}^{\gamma^{2}}$ & 3.62 & 3.66 \\
\hline
\end{tabular}

Note. Comparison of the distances observed in the C47S mutant and in the wild-type enzyme (lhd2).

The presence of a benzoate ion in contact with the active site residues has already been described in the crystal structure of chloroper-oxidase from S. aureofaciens Tü24 (pdb entry 1a8u) ${ }^{24}$ An organic acid is required in this enzyme for the haloperoxidase activity. The complex with benzoate was obtained after soaking the crystals in a solution containing sodium benzoate. However, the benzoate is not specific since complexes were also obtained with propionate and acetate. In these complexes, both carboxyl oxygen atoms of the organic acids are within hydrogen bonding distance from Ser98 $\mathrm{O}^{\gamma}$. Interestingly, these carboxyl oxygen atoms are also hydrogen bound to backbone nitrogen atoms, to His $257 \mathrm{~N}^{\varepsilon 2}$ belonging to the active site and to a $\mathrm{Thr} \mathrm{O}^{\%}$. Table 2a shows that this situation is very similar to our observations in PRDX5 (wild-type and C47S mutant) with the replacement of His $257 \mathrm{~N}^{\varepsilon 2}$ by $\operatorname{Arg} 127 \mathrm{~N}^{\eta 2}$ whose $\mathrm{N}^{\eta 1}$ interacts with the $\mathrm{S}^{\gamma}$ atom of Cys 47 (wild-type) or the $\mathrm{O}^{\gamma}$ atom of Ser47 (mutant). In PRDX5, it is however worth remembering that the benzoate ion was not added at any stage of the production, of the purification, or of the crystallization. However, we cannot completely exclude that the benzoate ion was present in bacterial culture media (LB Broth base including peptone 140 and yeast extract) used in the production of $E$. coli recombinant human PRDX5. The remaining contacts of the benzoate ion in PRDX5 involve its aromatic part and are thus mainly hydrophobic. The buried surfaces of the protein by the presence of this aromatic ring are very similar in the cases of the C47S mutant and of the wild-type enzyme, respectively $47 \AA^{2}$ in the C47S mutant compared to $52 \AA^{2}$ in the wild-type enzyme. These hydrophobic contacts between the protein and the benzoate ion concern four residues at the level of the protein and the shortest distances between these residues and the aromatic ring are given in Table 2b. As shown in Fig. 1, one residue, Thr147, appears on one face of the aromatic ring.

The three remaining residues (Leu116, Ile119, and Phe120), located on the other face, belong to the $\alpha 5$ helix which is a characteristic of PRDX5, since it is not present in other PRDXs structures. This $\alpha 5$ helix which appears as a bump at the surface of the protein thus seems to be an essential feature for holding the benzoate ion in its position. It constitutes one side of a kind of pincer in which the benzoate is locked, the other side being the Thr147 residue. When PRDX5 is oxidized, it was shown ${ }^{12}$ that the region 146-147 undergoes an important reorganization in order to bring the resolving residue Cys151 in the neighborhood of the peroxidatic residue Cys47 and to allow the formation of the disulfide bond. During this movement, one side of the pincer (Thr147) is thus relaxed and the benzoate ion can escape from the trap. From the comparison of nine independent molecules of PRDX5, the region of residues 146-147 was also recognized for its important flexibility. ${ }^{11}$

Interestingly, it was previously noticed ${ }^{12}$ that the $\alpha 5$ helix was involved in the formation of a non-covalent dimer between two PRDX5 molecules. This kind of dimer buries about $790 \AA^{2}$ per molecule and since it is present in structures of reduced and oxidized forms of PRDX5 belonging to different space groups and systems (tetragonal for $1 \mathrm{hd} 2,{ }^{10}$ monoclinic for $1 \mathrm{~h} 4 \mathrm{o},{ }^{11}$ orthorhombic for loc $3^{12}$ ) it is certainly not an artefact of crystallization. This dimer is also observed in the structure of the C47S mutant and is formed by the association of two molecules related by a crystallographic twofold axis. This association leads to the formation of a channel at the interface between the two molecules (Fig. 2) with the two benzoate ions obstructing both extremities of this channel. The benzoate ions appear as the guardians of the two gates giving access to the channel.

What could be the exact role of the benzoate ion in the mechanism of PRDX5 remains to be elucidated. Does it react with hydroxyl radicals produced during the enzymatic reaction to be decarboxylated ${ }^{13}$ or hydroxylated $?^{25}$ Does it undergo a nucleophilic attack on the carboxyl carbon, as in haloperoxidases? ${ }^{24}$ Is it involved in a 
completely different reaction? Or is its presence only fortuitous? This remains an open question.

Fig. 2. The benzoate ion appears at the extremities of a kind of channel at the interface of two molecules of the C47S mutant of PRDX5 associated in a non-covalent dimer. The side chain of Ser47 is shown. Figure prepared using MOLSCRIPT ${ }^{21}$ and RASTER3D. ${ }^{22}$

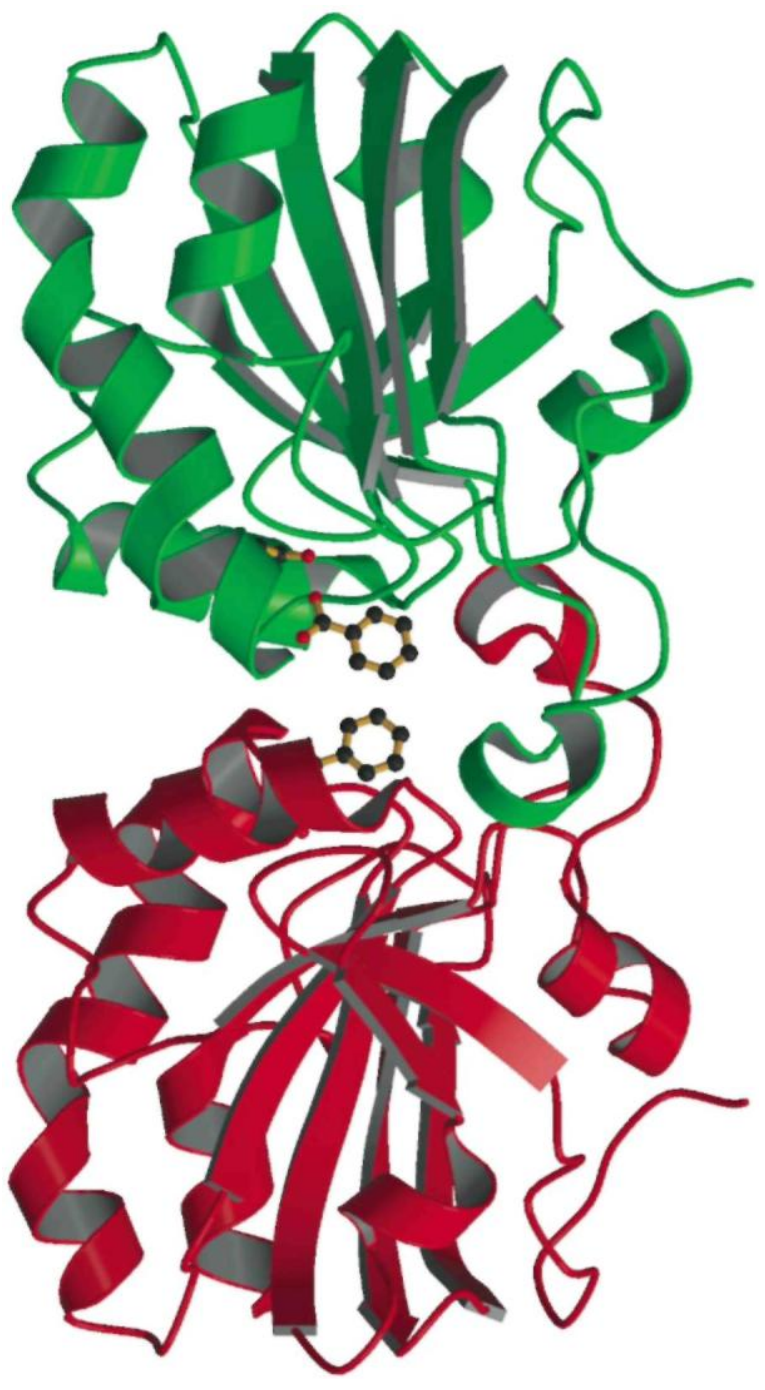

\section{ACKNOWLEDGMENTS}

This work was supported by grants from the "Fonds National de la Recherche Scientifique" (Belgium) and the "Communauté française de Belgique-Action de Recherches Concertées." We also thank the European Community for Access to Research Infrastructure Action of the Improving Human Potential Programme to the EMBL Hamburg Outstation, contract number HPRI-1999-CT-00017. We are grateful to the members of the scientific staff at the EMBL-Hamburg outstation for their help during data collection.

Supplementary material the coordinates and the structure factors have been deposited with the Protein Data Bank under accession code 1 urm. Further details of the crystal structure investigations may be obtained from http://www.rcsb.org/pdb/ or http://rutgers. rcsb.org/pdb/.

\section{References}

1. Rhee, S.G.; Kang, S.W.; Chang, T.S.; Jeong, W.; Kim, K. IUBMB Life 2001, 52, 35. 
Published in : Journal of Chemical Crystallography (2004), vol. 34, issue 8, pp. 553-558.

Status : Postprint (Author's version)

2. Wood, Z.A.; Poole, L.B.; Hantgan, R.R.; Karplus, P.A. Biochemistry 2002, 41, 5493.

3. Hofmann, B.; Hecht, H.-J.; Flohé, L. Biol. Chem. 2002, 383, 347.

4. Hirotsu, S.; Abe, Y.; Okada, K.; Nagahara, N.; Hori, H.; Nishino, T.S.; Hakoshima, T. Proc. Natl. Acad. Sci. U.S.A. 1999, 96, 12333.

5. Schröder, E.; Littlechild, J.A.; Lebedev, A.A.; Errington, N.; Vagin, A.A.; Isupov, M.N. Structure 2000, 8, 605.

6. Alphey, M.S.; Bond, C.S.; Tetaud, E.; Fairlamb, A.H.; Hunter, W.N. J. Mol. Biol. 2000,300, 903.

7. Choi, H.J.; Kang, S.W.; Yang, C.H.; Rhee, S.G.; Ryu, S.E. Nat. Struct. Biol. 1998, 5, 400.

8. Knoops, B.; Clippe, A.; Bogard, C.; Arsalane, K.; Wattiez, R.; Hermans, C.; Duconseille, E.; Falmagne, P.; Bernard, A. Biol. Chem. $1999,274,30451$.

9. Seo, M.S.; Kang, S.W.; Kim, K.; Baines, I.C.; Lee, T.H.; Rhee, S.G. J. Biol. Chem. 2000, $275,20346$.

10. Declercq, J.P.; Evrard, C.; Clippe, A.; Vander Stricht, D.; Bernard, A.; Knoops, B.J. Mol. Biol. 2001, $311,751$.

11. Declercq, J.P.; Evrard, C. Acta Crystallogr. 2001, D57, 1829.

12. Evrard, C.; Capron, A.; Marchand, C.; Clippe, A.; Wattiez, R.; Soumillion, P.; Knoops, B.; Declercq, J.P. J. Mol. Biol. 2004, 337 , 1079.

13. Sagone, A.L.; Decker, M.A.; Wells, R.M.; Democko, C. Biochim. Bwphys. Acta 1980, 628, 90.

14. Plaisant, F.; Clippe, A.; Vander Stricht, D.; Knoops, B.; Gressens, P. Free Rod. Biol. Med. 2003, $34,862$.

15. Otwinowski, Z.; Minor, W. Methods Enzymol. 1997, 276, 307.

16. Brünger, A.T. Nature 1992, 355, 472.

17. Murshudov, G.N.; Vagin, A.A.; Dodson, E.J. Acta Cryst. 1997, D53, 240.

18. Collaborative Computational Project, Number 4. Acta Cryst. 1994, D50, 760.

19. Jones,T.A.; Zou,J.-Y.; Cowan,S.W.; Kjeldgaard,M. Acta Cryst. 1991, A47, 110.

20. Perrakis,A.; Morris,R.M.; Lamzin, VS. Nat. Struct.Biol. 1999, 6, 458.

21. Kraulis, P.J. J. Appl. Cryst. 1991, 24, 946.

22. Merritt, E.A.; Bacon, D.J. Methods Enzymol. 1997, 277, 505.

23. Martin, J.L. Structure 1995, 3, 245.

24. Hofmann, B.; Tölzer, S.; Pelletier, I.; Altenbuchner, J.; van Pée, K.H.; Hecht, H.J. J. Mol. Biol. 1998, $279,889$.

25. Halliwell, B.; Grootveld, M.; Gutteridge, J.M.C. Meth. Biochem. Anal. 1988,33, 59. 